\title{
MedienPädagogik
}

Zeitschrift für Theorie und Praxis der Medienbildung

Themenheft Nr. 42: Optimierung in der Medienpädagogik.

Forschungsperspektiven im Anschluss an den 27. Kongress der DGfE

Herausgegeben von Patrick Bettinger, Klaus Rummler und Karsten D. Wolf

\section{Optimierung als digital-kapitalistisch}

Heidrun Allert

\section{Zusammenfassung}

Der Beitrag verortet die Optimierung des Individuums innerhalb einer digital-kapitalistischen Logik. Dazu betrachtet er die Lebensform ortsunabhängig lebender, selbstständig online arbeitender Menschen, die ihr Einkommen unter Verwendung datenbasierter, algorithmisch getriebener Plattformen generieren. Selbst nennen sie sich Digitale Nomaden. Der gefundene Zusammenhang von selbstermächtigendem Individualismus und Entstaatlichung lässt sich bis früh in die Netzwerkentwicklung zurückverfolgen. Der Beitrag basiert auf einer empirischen Studie in Form einer online Ethnographie. Die Untersuchung nimmt eine praxis-theoretische Perspektive ein. Sie betrachtet digitalen Kapitalismus nicht von der Analyse des Wirtschaftens grosser Konzerne oder der Äusserungen herausragender Unternehmensgründerinnen und -gründer, sondern aus der Sicht von Familien, Alleinerziehenden und jungen Erwachsenen, die individuelle Lösungen für herausfordernde Lebenslagen suchen und deren Individualismus politisch wird.

\section{Optimization as digital-capitalist}

\begin{abstract}
This paper describes socio-material practices of digital nomads who live locationindependent and work online. Their business models are based on data-driven platforms. Optimizing oneself as an individual is understood as a key element of a digital capitalist logic. Based on an empirical study, the paper outlines digital capitalism as co-produced by individuals who do no longer understand themselves as citizens of specific states. Its main ideas can be traced back to the beginning of the development of the World Wide Web and what is called "new economy".
\end{abstract}

\section{Einleitung}

In einer Instagram Story zeigt jemand seine Morgenroutine zu der das Training der Atemtechnik von Wim Hof gehört. Die Atemtechnik bereitet vor, sich in ein Becken zu legen, das mit Eiswürfeln und Wasser gefüllt ist. Ein Natural Bio-Hacking-PersonalTrainer verspricht, mittels Ernährung die Körperaktivitäten manipulieren und programmieren zu können; insbesondere den Schlaf, die Anpassung des Organismus an die Umgebung sowie die Aufmerksamkeit. Menschen berichten, dass sie auf Zucker

Allert, Heidrun. 2021. «Optimierung als digital-kapitalistisch». MedienPädagogik 42, (Optimierung), 301-326. https://doi.org/10.21240/ mpaed/42/2021.06.24.X. 
und allergene Lebensmittel verzichten, dass sie lange Fastenzeiten und sehr kurze Esszeiten einrichten, gewisse Mengen von Pilzen für mehr Ausdauer, Immunpower und Produktivität einnehmen, den Fokus schärfen, meditieren und den flowstate aktivieren. Sie verbinden sich mit ihrem Bewusstsein, begeben sich in Prozesse der Persönlichkeitsentwicklung, die ihnen helfen, gesellschaftlichen Erwartungen nicht mehr entsprechen zu müssen, gehen in die Eigenverantwortung und raus aus der Angst. Sie stellen ihre eigene Zukunft her, indem sie Entscheidungen treffen, ins Tun kommen, sich das Leben kreieren, das sie leben möchten und aufhören, ihre Visionen mit den Emotionen und Verhaltensmustern von gestern zu verknüpfen. Sie hinterfragen ihre alten Reaktionsmuster, Konditionierungen und Glaubenssätze und reprogrammieren ihre Gedanken. Wenn sie in ihrem Kopf hören «Das kann ich nicht!», versuchen sie es einfach zu tun. Sie streben an, der Mensch zu sein, der sie wirklich sind, die innere Wahrheit zu hören, sich nicht mehr einzuschränken oder zu limitieren. Sie verlassen ihr Hamsterrad, tauschen nicht mehr Zeit gegen Geld - sie nehmen Coachings wahr, um in die Selbständigkeit und von dort auf ein neues Level zu gelangen und als Unternehmer ein skalierbares, automatisiertes Business zu betreiben. Lernen, Wachstum und Persönlichkeitsentwicklung ergeben sich immer und überall, sobald sie aus ihrer Komfortzone gehen.

So sind Narrative ortsunabhängig lebender und online-selbständig arbeitender Menschen formuliert, die auf Instagram geteilt werden. Familien, Alleinerziehende und junge Erwachsene optimieren sich und beschreiben ihre Lebensform als «alternativ», bedürfnis- und familienorientiert. Wozu dient die Optimierung des Individuums hier und in welcher Logik erscheint dies stimmig? Die Praxisform wird von ihren Protagonistinnen und Protagonisten als individueller und selbstbestimmter Entwicklungsprozess gerahmt und wird damit politisch. Der vorliegende Beitrag ordnet die Optimierung des Individuums in dieser Praxisform zu einer Logik des digitalen Kapitalismus. Letzterer wird von Staab (2019) und Zuboff (2019) betrachtet als das Wirtschaften multinationaler Konzerne mit datenbasierten Geschäftsmodellen wie Amazon, Alphabet, Facebook und Zoom, bezeichnet auch als «Plattformökonomie». Nachtwey und Seidl (2017) rekonstruieren den Geist des digitalen Kapitalismus aus Äusserungen digitaler Eliten. Im vorliegenden Beitrag wird untersucht, wie digitaler Kapitalismus auch durch die Praxis von Individuen, durch geteilte Narrative und Legitimationsfiguren hergestellt und ausgebreitet wird. Sie wird von Individuen geteilt und prägt sich zu kulturellen Mustern und Praktiken aus. Diese emergenten Praktiken werden hier in ihrer Kontingenz skizziert. Obwohl die Praxisform von ihren Protagonistinnen und Protagonisten als Alternative zum Leben in der Gesellschaft gerahmt wird, wirkt sie in die Gesellschaft hinein.

Digitaler Kapitalismus ist also nicht ausschliesslich eine top down Entwicklung, nicht nur das Wirtschaften multinationaler Konzerne, die Monopol bildende Plattformen, Infrastrukturen und datenbasierte Geschäftsmodelle generieren. Er wird 
vielmehr auch von Individuen hergestellt und verbreitet: durch deren performative Praktiken der Lebensbewältigung, Alltags- und Beziehungsgestaltung sowie der Generierung von Einkommen.

Treibende Akteure des digitalen Kapitalismus behaupten, öffentliche Aufgaben wie Bildung, Gesundheit, Infrastruktur und Mobilität besser bewerkstelligen zu können als Staaten und staatliche Institutionen. Das reicht von einer Privatisierung von Aufgaben wie Kinderbetreuung, Bildung, Gesundheit und Absicherung bis hin zur expliziten Ablehnung staatlicher Institutionen, Regierungen und Gesellschaft. Die Ideologien des digitalen Kapitalismus entstanden nicht erst mit der zunehmenden Digitalisierung oder dem, was jüngst als «Solutionism» (vgl. Nachtwey und Seidl 2017, Morozov 2013) bezeichnet wird. Fred Turner (2006) zeichnet die enge Verknüpfung der Entwicklung und Ausbreitung des World Wide Web und der «New Economy» auf, startend mit Bewegungen in den 1960er Jahren und in Akteursnetzwerken der 1990er Jahre. David Gerard (2017) beschreibt die Ideologie, auf der etwa auch die Kryptowährung Bitcoin fusst, als libertären, rechtsorientierten Anarchismus, als «anarcho-capitalism» (ebd., 18). Dieser basiert auf dem Glauben der Überlegenheit von Eigentumsrechten und der kompletten Eliminierung des Staates. "Cyberlibertarianism» ist nach Gerard der akademische Begriff für den Strang dieser Ideologie im frühen Internet (Gerard 2017, 18). In diese Entwicklung des Netzes, die nie rein technisch, sondern immer auch politisch war, werden die Befunde der vorliegenden Studie in der anschliessenden Diskussion eingeordnet.

Der Beitrag basiert auf einer empirischen Studie und beschreibt Praktiken und Legitimationsfiguren wie sie von Familien, Alleinerziehenden und jungen Erwachsenen, die ortsunabhängig leben und online-selbständig arbeiten, auf ihren Social Media Accounts dargestellt werden. Die Ergebnisse der Studie werden als Personas und Befunde zusammengefasst und in einer Diskussion eingeordnet. Die Studie macht sichtbar, dass Praxisformen entstehen, die forcierte Individualisierung und Eigenverantwortung propagieren, auf privatwirtschaftliche Akteure der Plattformökonomie angewiesen sind, in denen unternehmerisches Denken, ökonomisches Wachstum und Persönlichkeitsentwicklung in eins fallen und die, obwohl sie als individuell gerahmt werden, hoch politisch sind. Diese Praxisform, in der Freilernen und die Ablehnung der Institution Schule bedeutende Rollen einnehmen, ist für die institutionell etablierte Erziehungswissenschaft und Bildungspraxis bisher wenig sichtbar geworden. Der Beitrag wird Praktiken aufzeigen, die sich über Digitalisierung ausbreiten, durch sie überhaupt möglich werden und deren Wurzeln bereits früh in der Netzentwicklung liegen. Sie ist nicht die einzige Entwicklung und nicht DIE Entwicklung der Digitalisierung sondern eine - in wesentlichen Momenten antidemokratische - Entwicklung. Sie lässt sich als Entwicklungslinie rekonstruieren und in ihrer Kontingenz aufzeigen. 


\section{Empirie}

Die Studie wählt einen praxis-theoretischen Zugang, basierend auf Schatzki (2012), Hörning (2004) und Shaffer (2006). Sie untersucht, welches Verständnis Praktikerinnen und Praktiker ihren Entwicklungsprozessen und Praxisformen zugrunde legen: "the ways of knowing, of deciding what is worth knowing, and of adding to the collective body of knowledge and understanding of a community of practice» (Shaffer, 2006, 223). Göttlich (2010) bezeichnet «Medien als Durchgangspunkte sozialer Praktiken» (ebd., 29). (Unterschiedliche) soziale Praktiken werden laufend reproduziert und entworfen:

"Medien sind [...] selbst bereits Ausdruck praktischen Bewusstseins und formieren gerade dadurch, praxistheoretisch gesprochen, als Durchgangspunkte der Entstehung sozialer und kultureller Praktiken und nicht lediglich als Kreuzungspunkte im Sinne von Techniken für die Vermittlung von Kommunikation.» (ebd.)

Die empirische Studie nimmt die sozio-materiellen Praktiken von Menschen in den Blick, die ortsunabhängig leben, online-selbständig arbeiten und ihr Einkommen auf oder unter der Verwendung sehr grosser algorithmisch betriebener Plattformen generieren. Selbst nennen sie sich digitale Nomaden. Sie sind sich der Feed-Algorithmen der Plattformen bewusst und nutzen sie, um die Sichtbarkeit ihrer Anliegen, Produkte und Services auf Plattformen zu erzeugen. Plattformen wie Amazon, AirBnB, Facebook, Pinterest, YouTube, Instagram, Zoom bieten Einzelnen Möglichkeiten, eine Soloselbständigkeit beziehungsweise ein Online-Business aufzubauen und Reichweite zu erzielen. Social Media Marketing, Netzwerkmarketing, Amazon FBA (Fulfilment by Amazon), Coaching und Beratung, Online Kurse, Content Creation sowie Blogging mit Werbepartnerschaften und Affiliate Programmen sind die vielleicht bekanntesten Modelle. Erleichtert wird der Einstieg in die Selbständigkeit durch das Modell der Geoarbitrage. Hier nutzen Einzelne einen Standortvorteil: Auf einem währungsstarken Markt wie Deutschland werden Dienstleistungen und Produkte angeboten, bei gleichzeitigem Aufenthalt in einem Land mit geringen Lebenshaltungskosten. An Orten, an denen die Internetverbindung gut ist und viele digitale Nomaden verweilen, sind Hotspots entstanden. Dort teilen sie untereinander «Hacks», d. h. Tipps zu Businessmodellen, Steuer- und Visafragen bzw. Regularien der Einreise, Lebensvorstellungen, Businessideen und Narrative und loten nach eigenen Worten «Möglichkeiten» aus. Gleichzeitig sind sie auf Plattformen wie Instagram und Facebook gut vernetzt. Sie leben zeitlich befristet an spezifischen Orten in Thailand, auf Bali, in Vietnam, Indien, auf Zypern, Sardinien, an ausgewählten Orten in Kolumbien, Brasilien, Costa Rica, Panama, Mexiko und vielen mehr. Die Tätigkeiten erfordern neben Laptop, Internet und Fotoausrüstung meist keine weiteren Arbeitsmittel. So wird etwa beim Geschäftsmodell Amazon FBA mittels Datenanalysen ermittelt, 
welches Produkt sich häufig verkauft. Dieses wird dann über Amazon gehandelt - direkt aus Fabriken (bevorzugt in Asien) bezogen und auf der Plattform Amazon ebenfalls angeboten. Die Produktdarstellung für dieses ausgewählte Produkt wird so optimiert, dass der Sortieralgorithmus das Angebot auf Amazon bei einer Suche weit oben listet. Dieses vertreibt der/die Online-Selbständige, wobei alle logistischen Prozesse und Aufgaben Amazon in seinen Zentren übernimmt. Der Vorteil des Soloselbständigen gegenüber einem lokalen Händler ist, dass dieser ein einziges Angebot optimiert (das Angebot des Produktes, das sich nach Datenanalyse zahlreich verkauft), was Händler mit seinem gesamten Sortiment nicht leisten können. AirBnB wiederum erlaubt, durch Datenanalysen den tagesaktuell erzielbaren Preis einer Unterkunft zu ermitteln. Dieser kann weit über der lokalen Miete liegen, zu der ein Objekt kurz bis mittelfristig von einer/m Soloselbständigen angemietet wurde. Auch hier ist das Wissen um den betreffenden Algorithmus entscheidend, um das Angebot im algorithmisch sortierten Listing weit nach oben zu bringen und somit gut sichtbar zu machen. Die Geschäfte werden auf währungsstarken Märkten gemacht. Zudem haben Anbietende auf deutschsprachigen Märkten aufgrund der Sprache weniger Konkurrenz als auf englischsprachigen.

Untersuchungsgegenstand der vorliegenden Studie sind die sozio-materiellen Praktiken ortsunabhängig lebender, online-selbstständig arbeitender Menschen, wie sie von diesen selbst auf Social Media Accounts artikuliert werden. Social Media Plattformen sind in diese Praktiken und Artikulationen ko-konstitutiv verwoben.

«Praktiken sind dann das Medium, durch das eine ausgeprägte soziale Koexistenz geformt wird, durch das Menschen in einen mehr oder weniger engen sozialen und zeitlichen Zusammenhang gebracht werden, obgleich sie je individuell fortschreiten. Solche Praktiken sind soziale Arrangements von Menschen, die aufeinander bezogene Handlungen vollziehen, sich damit in sozial und kulturell erwartbare und einsichtige Beziehungen zueinander stellen und dabei (doch) eigenständige Identitäten artikulieren. Je komplexer und tonangebender solche Praktiken ins Leben treten, desto eher verdichten sie sich zu Lebensmustern bzw. Lebensstilen, in denen sich räumliche und zeitliche Bündel von Handlungs- und Verstehensweisen verknüpfen» (Hörning 2001, 193f.).

Rekonstruiert wird die Verwendung von Technologien, die Deutungsmuster und geteilten Sichtweisen. Empirisches Material sind die Artikulationen auf Social Media Kanälen selbst, die nicht verstanden werden als blosse Repräsentation von Aktivitäten, die offline stattfinden, sondern als Praktik, die ebenfalls materiell und sozial vermittelt ist. Die Studie betrachtet die Artikulationen, die mit und durch die Medien vermittelt hergestellt werden. Kriterium der Auswahl von Social Media Accounts für die Studie ist, dass die Accountinhaberinnen und -inhaber ortsunabhängig leben, ihre Ansässigkeit in Deutschland, Österreich oder der Schweiz abgemeldet haben, 
online-selbstständig arbeiten und ihr Einkommen unter Verwendung datenbasierter Plattformen generieren. Obwohl in dieser Praxisform private Lebensführung, persönliche Freiheit und individuelle Bedürfnisse von ihren Protagonistinnen in den Vordergrund gestellt werden, bilden ihre Handlungsweisen soziale Praktiken und Muster aus. Diese haben eine kulturelle und politische Dimension.

In die Studie sind 51 Instagram Accounts sowie, falls vorhanden, zugehörige YouTube Videos, Blogbeiträge und Podcasts einbezogen. Die Instagram Accounts haben zwischen 1.500 und 38,9 Tsd. Followerinnen und Follower. Die Erhebung erfolgte über sieben Monate, von Februar bis August 2020. Das erhobene Material umfasst Instagram Postings, Stories und IGTV Videos, YouTube Videos, Podcasts und Blogpostings. Auditives und audiovisuelles Material wurde in Teilen transkribiert und Memos wurden angelegt. Die für die Datenerhebung eingerichteten Accounts weisen die Autorin als Untersuchende aus. Da das Material offen im Netz zur Verfügung steht, umfasst die Anonymisierung auch, Aussagen nicht zu zitieren, sondern paraphrasiert wiederzugeben. Die Paraphrasen sind im Text kursiv geschrieben. Das Material (Texte und Transkript) wurde inhaltsanalytisch ausgewertet und die Verbreitung zentraler Aussagen nachgezeichnet. Das Besondere der Auswertung liegt darin, dass prägnante Narrative stetig wiederholt und reproduziert werden. Etwa: «Raus aus dem Hamsterrad», «Glaubenssätze hinterfragen», «Freiheit» oder «Konditionierungen reprogrammieren». Es wurde tiefergehend untersucht, wie diese verstanden werden. Das Verständnis der Narrative, die inhaltliche Ausgestaltung, wird teilweise geteilt, teilweise differiert es. Auf Basis des Materials werden zudem Prozesse nachgezeichnet, etwa wenn im Laufe der Untersuchung eine Entwicklung von einer Online-Selbständigkeit in ein OnlineBusiness stattfindet.

\section{Personas}

Zunächst werden die Ergebnisse in Form aggregierter Personas präsentiert, zu der jeweils mehrere Accounts ausgewertet wurden.

\subsection{Alleinerziehende Weltreisende}

Monique ist mit ihrer Tochter Paulina seit März 2018 in der Welt unterwegs und online selbständig. Ihre Wohnung in Deutschland habe sie aufgelöst, das Inventar verkauft und verschenkt. Auch während des Corona Lockdowns und trotz der Rückholaktion deutscher Staatsbürgerinnen und Staatsbürger ist sie in Südostasien geblieben. Denn es habe Gründe gegeben, Deutschland zu verlassen, die immer noch existierten. Mit der privaten internationalen Krankenversicherung sei ausserdem nur ein 6-wöchiger Aufenthalt in Deutschland abgesichert. In Deutschland habe sie ihr Masterstudium abgebrochen. Die Gegend, in der sie wohnte, sei gentrifiziert, Freunde nach und nach 
fortgezogen, und so seien die lokalen Netzwerke weggebrochen. Als Alleinerziehende habe sie sich von der Gesellschaft, von Institutionen und Staat nicht verstanden und unterstützt gefühlt. Sie sei am Burnout gewesen. Mit ihrer bedürfnisorientierten Haltung zum Kind habe sie sich ausserdem immer wieder erklären müssen. Wege und Strategien seien im bedürfnisorientierten Lebensstil individuell. Kinderbetreuung, Studium und Jobs seien kräftezehrend gewesen. Das Kind wollte oft morgens nicht in die Kita. Komme man aber unregelmässig, würde man schräg angeschaut und ein freches, freies, wildes Kind würde schnell in eine Schublade (im Falle ihrer Tochter ADHS) gesteckt. In Südostasien gäbe es demgegenüber das drop-in System - man bezahle für die Tage, an denen das Kind in die Kinderbetreuung gehe. Das Kind wähle jeden Morgen, ob es den Kindergarten besuchen wolle, oder nicht. Sie wünsche sich, ortsunabhängig so viel zu verdienen, dass sie ihr Kind in Südostasien in eine 1:1-Betreuung zu einer Nanny geben könne. Leider seien die bisherigen Babysitterinnen nur kurzzeitig verfügbar gewesen. Monique postet oft Bilder ihres Kindes auf Instagram mit dem Titel «freies Kind» oder «freie Kindheit». Die Lebenshaltungskosten seien gering in Vietnam und Kambodscha. Monique möchte so lange reisen, bis sie einen Ort für sich und ihre Tochter gefunden habe, an dem sie sich wohl fühlten. Das sollten drei oder vier Bases an verschiedenen Orten der Welt sein. Sie sei ortsunabhängig tätig. Neben dem Schreiben von Content für Websites anderer, eine Tätigkeit die pro Wort bezahlt würde, dem Pflegen von Social Media Accounts für andere Selbstständige, baue sie ihr eigenes Coaching-Angebot auf. Zunächst habe sie zum Thema «Bedürfnisorientiertes Begleiten von Kindern» gecoacht, ihr Herzensprojekt, nun auch zum Thema «Human Design». Human Design sei eine Typen-Lehre vom Menschen. Zunehmend würden Angebote zu diesem Thema entstehen, aber sie plane immer schon das nächste Projekt. Auf Instagram ist oft auch ihre Tochter zu sehen. In den Postings bespricht sie ihre Coaching-Themen. Sie habe mittlerweile ein eigenes Netzwerk aufgebaut: private Kontakte, berufliche Angebote und Aufträge sowie der Austausch über Einreisebestimmungen, Visa usw. Man sei immer auf der Suche nach Möglichkeiten, die sich kurzfristig ergeben. Aufgaben wie die Erstellung inrer Website vergebe sie an andere im Netzwerk. Sie treffe sich online und weltweit mit reisenden Alleinerziehenden und Familien und interessiere sich für die Themen Freilernen und schulfrei Leben. Leene (9), die Tochter einer Familie, mit der sie die Wintermonate auf einer Insel in Thailand in einem Resort verbracht hat, dürfe in jedem Land wählen, ob sie eine Schule besuchen möchte oder nicht. Das seien private Montessorischulen, Waldorfschulen und Internationale Schulen. Freilerner-Familien würden den Schulbesuch ermöglichen, wenn die Kinder den Wunsch danach äusserten. Immer wieder in Gemeinschaft zu leben, sei ihr wichtig. Üblicherweise verbringe sie mit mehreren Familien und Alleinerziehenden die Wintermonate gemeinsam in einem Resort auf der Insel Koh Phangan in Thailand. Manche seien immer wieder dabei, andere Familien zum ersten mal während einer Elternzeitreise. Die Kinder 
verbrächen dann viel Zeit zusammen und die Betreuung könne aufgeteilt werden. Da finde viel soziales Lernen statt und die Älteren nähmen die Kleineren oft mit zum Spielen, was ihre hohe soziale Kompetenz zeige. Eine ihrer weltreisenden Freundinnen lebe mit ihrem Mann von Bitcoin Mining und Beratung. Da sei das Familienmodell ganz klassisch. Sie betreue die Kinder und kümmere sich um den Haushalt, er baue das Online-Business auf. Monique sagt, sie folge ihrer inneren Wahrheit. Ihre Wahrheit sei, was mit ihr räsoniere. Selten nehme sie Stellung zu politischen Themen. Etwa zu Corona. Sie habe auf ihrem Instagram Account die Demo in Berlin am 29.08.2020 gegen die Corona-Massnahmen verteidigt. Die unzähligen Nachrichten zu ihrem Posting, teils herzlich, teils kritisch, möchte sie nicht beantworten, die Zeit solle lieber ihrer Tochter zu Gute kommen. Sie spreche nur ihre Wahrheit aus dem Herzen. Man könne die eigene Wahrheit spüren, denn sie sei eine Energie und damit real. Üblicherweise erlebe sie ihre Instagram Follower (über 10.000) als unterstützend. Viele schreiben, sie seien inspiriert von ihrem Mut und Lebensstil. Sie selbst wolle inspirieren, wer nicht inspiriert sei, könne jederzeit entfolgen. «Mein Account, meine Regeln». Als ihre Tochter ins Schulalter gekommen sei, habe sie sich und ihr Business ganz aus Deutschland abgemeldet. Sie habe keinen Wohnsitz in Deutschland, sie sei dort steuerfrei. Sie bleibt deutsche Staatsbürgerin. Das Leben sei schön, wenn man gesund, ganz und vollkommen sei, ohne auf jemanden angewiesen zu sein. Sie absolviere Onlinekurse und investiere in sich selbst. Derzeit Dr. Joe Dispenzas Kurs: Redesigning your Destiny, der auf dessen Website beworben wird:

"In diesem aufregenden neuen Onlinekurs macht dir der New York Times-Bestsellerautor Dr. Joe Dispenza die dir innewohnende Kraft bewusst, mit der du jeden Aspekt deines Lebens verändern kannst - von der Zellebene bis hin zu deinem Lebensplan.»

Themen sind Neuroplastizität, Meditation, Energiearbeit mit dem Ziel, die eigene Realität zu kreieren (https://drjoedispenza.de).

Müsse sie sich schuldig fühlen, weil sie nicht erst das System repariert habe, bevor sie den Bedürfnissen ihres Kindes nachkomme? Nein! Dass in Deutschland so magische Themen wie Menschsein so rational besprochen werden, falle ihr schwer. Jede Magie werde ausgeblendet. Mehr und mehr vertraue sie ihrer Intuition. Die Gesellschaft konditioniere uns, aber man könne das alles reprogrammieren. Ihr gehe es so viel besser, seit sie ihre Masken fallen lassen könne, keine gesellschaftlichen Erwartungen und Rollen mehr erfüllen müsse, ihre Sinnlichkeit lebe, bewusst sinnliche Erfahrungen mache, ganz bei sich und im Moment sei, sich selbst bewusster wahrnehmen würde. Wenn sie ins Jetzt sinke, Embodiment praktiziere, dann habe das, was sie tue, mehr Qualität. Die Gesellschaft habe ihr eingeredet, dass man etwas leisten müsse. Sie habe versucht, sich als Frau zu behauten, aber in Wissenschaft und Unternehmen hätten Frauen es immer noch schwer. Ein Online-Business aufzubauen, 
da würden ihr weniger Steine in den Weg gelegt und sie sei jetzt erfolgreich. Sie fühle sich immer noch schuldig, wenn sie Pausen mache. Es habe viel Kraft gekostet, in einer Gesellschaft zu bestehen, die oft kein Verständnis für eine andere Lebenseinstellung zeige. Was sie sich aufgebaut habe, habe sie durch eigenen Fleiss und durch ihre Selbständigkeit erreicht. Aber Menschen seien unterschiedlich leistungsfähig. Man sei auch ohne Leistung wertvoll und wenn man für sein Kind Bedürfnisorientierung ermögliche, dann sollte man sich das auch selbst zugestehen. Man müsse sich Pausen und das freie Leben nicht verdienen. Sie teile sich den Tag so ein, dass es zu ihrer Energie passe und habe den Fokus auf ihr Kind und ihr Business gelegt.

\subsection{Reisefamilien}

Andreas, der Vater einer fünfköpfigen Familie, Online-Unternehmer und MindsetCoach sagt, alle Informationen zum Aufbau einer Online-Selbständigkeit fänden sich im Netz. Worum es aber wirklich gehe, sei, eine Entscheidung zu treffen. Auch der Preis seines Coachings erfordere eine Entscheidung. Raus aus dem System, rein in die Eigenverantwortung. Es gehe um die Arbeit am Mindset. Es gelte, die Glaubenssätze der Gesellschaft und der vorausgehenden Generationen zu hinterfragen. Frauen seien in der Gesellschaft gedrängt worden, einen männlichen Alltag zu leben. Im Beruf erwarte man von ihnen, so zu tun, als hätten sie keine Kinder zuhause. Auch ihren monatlichen Zyklus dürften Frauen im beruflichen Alltag nicht wahrnehmbar machen, obwohl es da Phasen des Schaffens und des Rückzugs gebe. Die Rücksicht auf den Zyklus bringe mehr Energie. Ihr Grund, Deutschland zu verlassen, sei der Wunsch beider Partner nach mehr Familienzeit gewesen. Die Schichtdienste in ihren Gesundheitsberufen seien nicht mit der Betreuung der Kinder vereinbar gewesen. Zunächst hätten sie einen Onlinekurs zum Thema «Vaterschaft und Elternzeit» aufgebaut, heute sei er Mindest-Coach und Freedom Warrior. Sie lebten im Winter in Thailand, Mexiko oder Costa Rica, im Sommer in Europa. In Thailand lebten sie meist in einer Villa mit Gemeinschaftspool, in Europa in einem Campervan, in Südamerika in AirBnB-Wohnungen. Mit dem Einblick in sein privates Leben wolle er einen Mehrwert bieten. Er vertraue nicht mehr auf die staatliche Rente. Als Angestellter mit 2.400 netto habe ihn das belastet. Jetzt sei das kein Thema mehr, denn er habe investiert in Assets, Bitcoin, Gold und P2P Investments auf entsprechenden KreditvermittlerPlattformen. Derzeit starte sein neuer Coaching-Kurs zum Thema: «Die Online-Selbständigkeit auf das nächste Level heben - Online-Unternehmertum durch Automatisierung». Kundinnen und Kunden lebten in Österreich, Deutschland, der Schweiz und weltweit ortsunabhängig. Die beiden Online-Unternehmen, sein eigenes und das seiner Frau, seien in Malaysia angemeldet. Für die Anmeldung seien sie eine Woche lang in Kuala Lumpur gewesen. Das sei kein grosser Schritt mehr, wenn man selbst Berater und Coaches habe, die da Vorreiter sind. Mit Coachings investiere er in sich selbst und in sein Mindset. 
Der gemeinsame Alltag sei gut strukturiert und werde immer wieder zwischen den Ehepartnern abgesprochen. So bewältigten sie die Betreuung der Kinder, die Arbeit, ein herausforderndes Fitnesstraining sowie Paarzeit. Langfristig würden sie nicht planen. Sie hätten sich 2017 aus Deutschland abgemeldet, das Haus verkauft, lebten zunächst in ihrem Campervan und reisten durch Europa. Sie hätten nach und nach ihr altes Leben, alle Erwartungen und Glaubenssätze losgelassen und seien bereit für Neues geworden. Es sei eine neue Lebenseinstellung. Noch nie hätten sie sich vorher so frei gefühlt. Sie hätten verstanden, dass Freiheit aus einem selbst heraus entstehe, wenn man sich selbst akzeptiere und nicht mehr anpasse. Die Kinder seien Freilerner, aber nie alleine. Sie träfen immer wieder auf andere Familien; für den Winter verabredeten sie sich regelmässig mit anderen. Die Frage nach der Finanzierung des Lebensstils, nach der Schulpflicht und den späteren Chancen der Kinder erhielten sie oft auf Instagram. Schulpflichtig seien die Kinder nicht mehr, seit die Familie sich aus Deutschland abgemeldet habe. Zunächst sei das eine rein pragmatische Entscheidung gewesen. Einen Schulabschluss könne man auch extern machen. Das sei aber auch nicht mehr unbedingt nötig. Google, Amazon und andere innovative Unternehmen machten es bereits vor und so laufe das in Zukunft - die Unternehmen hätten Assessment Center und fragten nicht mehr nach einem Schulabschluss. Es könne aber sein, dass grade für Kinder mit Migrationshintergrund in Deutschland Schule wichtig sei. Aber auch für diese müsse sich Schule verändern. Seine Frau und er würden eine Stiftung gründen wollen zu einem Thema, das ihnen wirklich wichtig sei.

Man könne aus den gesellschaftlichen Regeln ausbrechen. Es sei wichtig, in die Eigenverantwortung zu gehen. Sicherheit und Stabilität lägen nur in ihm selbst, nicht im Aussen, man könne sie nur selbst herstellen. Mit Liebe, Vertrauen ins Leben und die eigene Leistungsfähigkeit gehe man mit der Unsicherheit der Welt um. Das brauche Mut und Wollen. Vieles sei nicht mehr, wie es mal ausgesehen habe. Sie lebten bewusst, gesund und kümmerten sich, versorgt zu sein. Er verlasse sich nicht mehr auf ein System, sondern nur noch worauf er Einfluss habe. Er arbeite an Fitness, Mindset und seinem Inneren. Andere könnten anders entscheiden und es sei gut, dass Menschen ihren Platz gefunden hätten und dort glücklich seien, etwa als Ärzte in einem Krankenhaus. Ansonsten könnten auch sie etwas ändern. Es gelte, die eigenen Werte nach aussen zu bringen. Alles sei schon in seinen Klienten vorhanden - es sei ihnen nur noch nicht gesagt worden. Wenn man abhängig vom Aussen sei, dann schaffe man es nicht. Es habe ihn nicht ausgefüllt, was er gearbeitet habe. Er wollte nicht nur stabil halten, sondern wachsen, sein Potential erhalten und andere Menschen berühren und unterstützen. Jetzt gäbe es mehr Höhen und Tiefen. Nur seiner Familie habe er ein Versprechen gegeben. Es brauche ein Mentoring um diesen Weg zu gehen. Sein Online-Programm würde von Menschen mit Expertise in Gesundheitsberufen, erzieherisch-pädagogischen Bereichen und der Finanzberatung gebucht. Er habe gelernt, 
örtlich völlig unabhängig Geld zu verdienen und zu verkaufen: Menschen darin zu unterstützen, endlich in ihre Kraft zu kommen und online durchzustarten. Das sei so eine abgefahrene Freiheit für sie als Familie. Sie beschenkten sich mit ihrer erfolgreichen, sinnstiftenden Arbeit jeden Tag selbst. In aller Grossartigkeit könne das jeder - alles stecke in einem. Das Unterbewusstsein bestimme das Vermögen.

Es gehe darum in die Freiheit zu gehen. Freiheit sei eine Lebensart. Äussere Freiheit könne der Startpunkt sein: den Job und Verträge kündigen, das Haus verkaufen und den Haushalt auflösen. Zeit nicht mehr gegen Geld zu tauschen. Die wirkliche, innere Freiheit komme aus der Motivation, sich selbst freizusetzen von beschränkenden Glaubenssätzen. Die Überforderung innerhalb der Gesellschaft liege in den vorhandenen Rollenmodellen, zum Beispiel zur Vaterschaft, Männlichkeit und Partnerschaft. Das ist der Startpunkt eines möglichen Coachings, um die begrenzenden Elemente des bisherigen Lebens zu finden. Man könne zu den Wurzeln gehen, Beschränkungen auflösen, um das authentische Selbst zu finden. Man profitiere von einem Werkzeugkasten, der zur eigenen Freiheit führe wie Körperarbeit, Bewegung, Atemarbeit, Meditation und Barfusslaufen.

\subsection{Die Vorreiter}

Martin ist mit seiner Partnerin Sandra seit 2014 weltweit unterwegs. Die Zeit im Schweigekloster in Thailand und beim Yawanawa Stamm im brasilianischen Amazonas Dschungel sei wichtiger Teil des Prozesses und der Transformation gewesen. Auf dem Weg solle es weiter gehen, immer wieder offline zu sein und andere Bewusstseinsformen kennenzulernen. Was wir heute erlebten, sei der Kollaps eines alten Systems, das auf Angst, Mangel, Gier und Kontrolle aufgebaut sei. Es existierten weisere Kulturen als unsere eigene: Indigene Völker, die mit einem unbeschreiblich ausgereiften Verständnis der Zyklen von Zeit und kosmischen Kräften ausgestattet seien, die um vielfaches mächtiger seien als korrupte Regierungen und Konzerne. Er nehme das neue Paradigma an - dies alles habe Teil am Aufbau einer neuen Zukunft. In den kommenden Jahren und Jahrzehnten möchte er im höchst möglichen Flow sein und kreieren. Wenn man heile von den Erlebnissen und Glaubenssätzen vorausgegangener Generationen, den Traumata und Erwartungen der Gesellschaft, dann würden die eigenen Kreationen besser werden.

Verantwortung könnten wir nicht im Aussen suchen. Man könne sich fragen, ob man selbst in Integrität lebe, ob man Sachen kaufe, die nachhaltig hergestellt wurden, ob man selbst Sorge dafür trage, der Umwelt nicht zu schaden, ob man sein Business auf Kosten anderer mache, ob man aufrichtig und ehrlich zu anderen sei, ob man seine Wahrheit spreche und mit seiner Sichtweise rausgehe. Sie selbst seien bereit für eine dezentralisierte Gesellschaft und Eigenverantwortung. Alte Machtsysteme würden zerfallen. Die neue Welt würde eine andere Version seiner selbst 
erfordern. Entrepreneure würden helfen, die neue Welt zu kreieren, Business Owner würden fairer an alle bezahlen, die an Projekten beteiligt seien, statt Hierarchien, gebe es Ko-Kreation. Es sei entscheidend, die Kontrolle über seine Gedanken zu haben. Die eigene Aufgabe sei, sich zu erinnern, wer man war, bevor die Welt einem erzählt hat, wer man sein solle. Ihr Weg müsse nicht für andere richtig sein. Nur sein Herz, kein Experte, keine Regierung, keine Kirche könnte sagen, was man tun solle, alles habe eine Ordnung, einen Sinn. Liebe, Wahrheit und Freiheit seien die Tür zur neuen Welt, während die alten Machtsysteme an der alten Welt klammerten. Er nehme die Unsicherheit an, mache seine eigenen Regeln und wähle Freiheit.

Nicht das chronologische Alter zähle, sondern das biologische, die Summe aus kalendarischem Alter sowie körperlichen und seelischen Einflüssen. Dieses liesse sich durch den Lebensstil beeinflussen. Man müsse stressvolle und ängstliche Gedanken loslassen. Er nutze immer jede Möglichkeit, sich zu qualifizieren und etwas zu lernen. Er habe spontan den Tauchschein gemacht, den Sportbootführerschein und Kampfsportarten. Mit kleinen Transaktionen bereite man sich auf grosse vor, und stetig lerne er dazu mit Podcasts, Büchern und damit einfach loszustarten. Noch bevor er ein Grundstück habe kaufen wollen, habe er sich für Grundstücke interessiert und sich rumführen lassen, zum Beispiel auf Borneo.

Durch Geschäftsmodelle wie Amazon FBA und der weltweiten Anmietung von Wohnungen in Brasilien, Kolumbien und Thailand und deren Vermietung auf AirBNB, mit Online-Kursen auf der Plattform Kajabi, mit Business Beratung, Coaching zu Biohacking, der Vernetzung der deutschsprachigen Community ortsunabhängig lebender Menschen seien er und seine Freunde finanziell frei. Finanzielle Bildung sei heute wichtig. Er fliege zu Beratern wie Tony Robbins, einem Trainer des Neuro-Linguistischen Programmierens (NLP) und Begründer der Neuroassoziativen Konditionierung. Dort in den vorderen Reihen zu sitzen und first class zu fliegen, sei wichtig, um unter Gleichgesinnten zu sein. Er lese viel und schnell und könne Bücher empfehlen wie «The Inner Engineering» von Sadhguru oder «Digital Nomad» von Makimoto und Manners aus dem Jahr 1997. Da wird das Phänomen ortsunabhängigen Arbeitens schon vorausgedacht. Ein faszinierendes Buch sei ausserdem «Grow a New Body How SPIRIT and Power Plant NUTRIENTS Can Transform Your Health» von Dr. Alberto Villoldo. Es gehe um die Aktivierung der heilenden Kraft von innen, das Hacken und Reprogrammieren des Körpers und der DNA. Die Natur habe eigentlich kein Interesse an der Langlebigkeit von Individuen, aber der Einzelne habe es. Das industriell hergestellte Essen mache uns krank, die Jobs stressten und überall seien Toxine. Es gehe aber darum, richtig gesund zu sein. Der Geist könne den Körper heilen. Schamanische Praktiken und cutting-edge Wissenschaft träfen da aufeinander.

Er habe immer viel gearbeitet. Deshalb lasse er immer wieder Altes los und baue etwas Neues auf. Sein Einstieg sei Tim Ferris' Buch «Die vier Stunden Woche» gewesen. Das Modell Geoarbitrage heisse, Globalisierung auf das Individuum zu beziehen, 
für sich selbst zu nutzen, was Unternehmen tun. Er habe vor Jahren ein Studium abgeschlossen und Konsumschulden gehabt. Im Studium hätte für ihn vieles keinen Sinn gemacht. Auf der Plattform Udacity habe er sich in neue Themen eingearbeitet, auch in das Geschäftsmodell Amazon FBA. Jahrelang seien sie mit Touristenvisa um die Welt gereist. Um nun aber länger in Ländern wie Brasilien, Panama und Thailand zu verweilen, hätten sie investiert. 50.000 EUR in Brasilien zu investieren oder ein Business in Thailand einzukaufen, ermögliche, mit Businessvisa einzureisen und länger zu bleiben. Sein jetziges Business sei in Kanada angemeldet wo Buchhaltungspflichten und Steuern für online Geschäfte gering seien und man nicht ansässig sein müsse. Auch die e-Residency in Estland sei interessant. Jahrelang sei er minimalistisch gereist. Sharing sei noch immer sein Credo. Aber jetzt gehe es auch um Assets und um Investitionen. In Aktien investiere er nicht, denn das System sei immer noch auf FIAT-Geld aufgebaut. Die FED erschaffe Geld aus dem nichts. Es sei an der Zeit, FIAT-Währungen gegen Gold gedeckte Währungen abzulösen. Regelmässig lege er in Bitcoin an, da gehe es nicht um Spekulation, sondern um eine Alternative zum Geldsystem, das immer noch auf Regierungen beruhe. Bitcoin würde midterm und longterm die Welt verändern. Der tatsächliche Wert des Bitcoin stehe fest, sobald alle damit bezahlten. Zu einer Immobilie in Deutschland rate er nicht, da man dann in die beschränkte Steuerpflicht rutsche. In Brasilien hätten sie ein Grundstück an einem Strand gekauft und mit lokalen Kräften eine kleine Villa gebaut. Der Ort sei ideal zum Kite-Surfen. Wenn sie nicht da seien, würde sie vermietet und Freunde seien immer willkommen. Es solle eine Base für alle sein. Es sei wichtig, mit lokalen Anwälten zu arbeiten, die das alles sicher machen, sonst habe man manchmal nur gepachtet, weil es verschiedene Vertragsformen gebe. Gleichzeitig bauten sie in Thailand ebenfalls eine Villa auf einem Seaview-Grundstück, das noch erschlossen werden müsse. Kriterien für seine weiteren Homebase-Orte seien: Community, Meer, Insel, Natur, tropisch, ganzjährig warm, Sport-Möglichkeiten, Kitesurfen, Gym, Yoga, gesundes veganes Essen, Wifi. Die ganze Welt stehe ihnen zur Verfügung.

Nationalstaaten und Grenzen würden derzeit noch aufrecht erhalten. Eine von deep state getriebene Politik, um den Prozess des individuellen und planetarischen Aufstiegs zu vermeiden, in den wir eingetaucht seien. Die Welt trete in eine neue Frequenz ein. Im great awakening würden die Menschen, die das entsprechende Bewusstseinsniveau haben, den Shift der Welt in ein neues Energiestadium spüren. Man könne in der Natur trainieren, das beruhige das Nervensystem, ohne Angst und im Vertrauen in das Leben sein, meditieren, in den Bergen wandern, sich von äusseren Einflüssen schützen, nicht in die alten Strukturen der Angst gehen. Unsere Schwingungen setzten sich zusammen aus unseren Gedanken, Glaubenssätzen, Emotionen und Gefühlen. Das Universum antworte auf unsere Schwingungen. Deshalb sei es so wichtig sich klar zu werden, welche Schwingungen man aussende. Alle Menschen seien gleich und hätten den Wunsch, Authentizität zu leben. Es müsse keinen 
Konkurrenzkampf geben, denn es gebe im Universum für jeden genug. Durch Achtsamkeit, Selbstfürsorge, Meditation und bestimmte Pflanzen liessen sich höhere Bewusstseinszustände erreichen. Man solle ein Business nicht aus Angst starten, sondern aus der Energie heraus der Menschheit einen Dienst zu erbringen und schwerwiegende Probleme für andere zu lösen.

\section{Befunde}

In den dargestellten Narrativen wird auf die Natur des Menschen rekurriert. Das Potential des Individuums werde durch die Gesellschaft, durch Tradition und Sozialisation begrenzt und könne entfaltet werden durch spirituelle und unternehmerische Praktiken. Die jeweilige Unternehmung sei ein Herzensprojekt, das Sinn mache und Probleme löse. Durch Technik und Optimierung kann in dieser Logik der Naturzustand überstiegen werden. Krise und Ambivalenz werden negiert zugunsten unhinterfragbarer Wahrheiten. Identität liegt in letztendlichen Ursprüngen und Archetypen, Sicherheit innerhalb der eigenen Person. Selbst Intuition kann optimiert werden.

Trotz dem Beharren darauf, dass jeder seinen Weg gehen und seine eigenen Entscheidungen treffen könne, ist diese Lebensform politisch. Narrative und Legitimationsfiguren werden geteilt und kulturelle Muster ausgeprägt. Dieser Abschnitt zeichnet die Logik dieser Praxisform nach. Sie existiert nicht als der Praxisform vorausgehend, sondern wird durch sie erzeugt. Die kursiv geschriebenen Aussagen sind paraphrasierte Zitate aus dem empirischen Material.

\subsection{Die Sphären Privat, Öffentlich und Ökonomisch fallen in eins}

Oftmals entsteht ein Online Business aus persönlichen Bedürfnissen, Erfahrungen und Kenntnissen: Die Kenntnisse aus der Finanzierung der Reise mit Bitcoin wird überführt in ein Online Kursangebot mit Beratung zum Thema Bitcoin, die Orientierung an bedürfnisorientierter Erziehung mündet in ein entsprechendes Coaching, die Erfahrung der Fremdbestimmung in der ersten Schwangerschaft zu einem Kurs zur Selbstbestimmung in der ersten Schwangerschaft, die Alleingeburt einer Medizinerin zu einem Kurs über natürliche, angst- und schmerzfreie Geburt. Die Businessangebote zielen auf die Problemlösung für Individuen.

In dieser Praxisform werden einfache Dichotomien propagiert. Etwa: Gesellschaft, gesellschaftliche Erwartungen und Rollen werden natürlicher Identität und innerer Wahrheit gegenübergestellt. Die Ablehnung von gesellschaftlichen Rollen soll den Menschen zu sich selbst als natürlicher Entität führen können. Die eigene Arbeit soll völlig mit dem eignen Selbst abgestimmt sein, rät eine Ratgeberin auf Instagram. Persönliches Wachstum wird mit ökonomischem gleichgesetzt. Zunehmend wird das «System Human Design» präsentiert, demzufolge jeder Mensch mit einem 
festgeschrieben Design auf die Welt kommt. Somit sind Charaktereigenschaften, Typen und Bedürfnisse festgeschrieben, eindeutig erkennbar und erfüllbar. Erziehung erscheint obsolet. Sozialisation bedeutet Konditionierung, die reprogrammiert werden könne.

Die Sichtbarkeit auf Social Media Plattformen ist in dieser Praxisform eine zentrale Form, als Subjekt in Erscheinung zu treten, insbesondere wenn staatlich oder institutionell zugesicherte Formen abgelehnt werden. Um Sichtbarkeit und Reichweite zu erzeugen, ist ein Wissen um die Sortieralgorithmen der News-Feeds zentraler Plattformen erforderlich. Die Form des «in Erscheinung Tretens» wird durch die Plattformen mitbestimmt. Immer wieder wird über das Sichtbarmachen der eigenen Kinder diskutiert. Würden sie nicht gezeigt, so würde es so aussehen als existierten keine Kinder in dieser Welt. Das Posten der Bilder erscheint nicht nur als Kalkül (etwa im Sinne des Ausbaus von Reichweite und der Darstellung der Themen und Kompetenzen des eigenen Online-Business), sondern auch als Alltagspraktik. Die Sichtbarkeit der eigenen Kinder wird teilweise aber auch kritisch diskutiert. Manche Accounts zeigen die Gesichter der Kinder nicht.

Postings erfolgen kontinuierlich oder auch vermehrt zeitgleich zur Bewerbung von Businessangeboten, die immer auch mit der eignen Lebenslage in Verbindung gesetzt werden.

\subsection{Entstaatlichung, Spiritualität, Individualismus und Optimierung}

Die empirische Studie zeigt, dass Kritik und Misstrauen in den Staat, seine Institutionen und Repräsentanten geäussert und hergestellt werden. Individuelles Bewusstsein löst in dieser Logik alte Machtsysteme ab. Bildungsinstitutionen und Institutionen der Kinderfremdbetreuung werden für die eigene Entwicklung oder die Bildung zumindest der eigenen Kinder für obsolet oder inadäquat erachtet - und das trotz hohem Interesse an stetigem Lernen, Persönlichkeitsentwicklung und Weiterbildung. Immer wieder wird das fehlende Vertrauen in staatliche Renten- und Finanzsysteme benannt. Entwicklungen wie Krypto-Währungen werden begrüsst, weil diese in ihrer Konstruktion selbst nicht auf das Vertrauen in staatliche Institutionen oder andere zentrale Instanzen angewiesen sind, sondern auf der Blockchain Technologie, d. h. der Nachvollziehbarkeit jeglicher Transaktion basieren und Rechenleistung als inneren Wert besitzen. Den FIAT-Währungen (Geld, welches durch die Zentralbank eines Landes ausgegeben wird) wird Vertrauen abgesprochen. Personen, die ihren Aufenthalt beziehungsweise Wohnsitz in Deutschland abmelden, bleiben deutsche Staatsbürgerinnen und Staatsbürger, bezahlen als Person keine Einkommenssteuer, die Kinder unterliegen keiner Schulpflicht. «Weltbürgerin zu sein», formuliert eine Digitale Nomadin. In der Regel basiert die Online-Selbstständigkeit auf Waren- oder Dienstleistungsangeboten innerhalb währungsstarker, insbesondere 
deutschsprachiger Märkte, wobei die einzelne Unternehmung bevorzugt in Malaysia, Kanada oder Zypern angemeldet ist, wo Steuern und Aufwand für Buchhaltung für ein Online-Business gering sind. Die Ablehnung geht in einigen Fällen bis hin zur Aussage, der Staat, bzw. seine Repräsentantinnen und Repräsentanten übten über Angst Kontrolle aus und manipulierten. Das fehlende Vertrauen in staatliche Absicherung, staatliche Konstrukte und Institutionen sowie die Ablehnung von Gesellschaft bedingt in dieser Logik die individuelle Absicherung und Eigenverantwortung. Diese zielt auf Optimierung, finanzielle Freiheit, spirituelle Praktiken, körperliche Fitness, Gesundheit, auf die Erlangung von spezifischen Bewusstseinszuständen sowie auf Persönlichkeitsentwicklung und stetiges Wachstum. Alternativ zu finanzieller Freiheit wird auch der Aufbau gemeinschaftlicher Lebensformen mit gegenseitiger Versorgung an verschiedenen Orten der Welt angestrebt. Propagiert wird ein forcierter Individualismus mit selbst gewählter Gemeinschaftlichkeit, bei gleichzeitig expliziter Ablehnung jedweder gesellschaftlicher und staatlicher Strukturen. Soweit spirituelle Angebote (bzw. deren Rezeption) das Individuum zum Ausgangspunkt der Veränderung der Welt ansehen, sind diese hoch anschlussfähig, eine Welt zu denken, die ohne Staaten bzw. ohne gesellschaftliche und staatliche Strukturen auskommt. Die Fokussierung auf das Individuum ist gemeinsamer Nenner des Spirituellen und der Ablehnung staatlicher Konstrukte. Gleichzeitig werden spirituelle Angebote herangezogen, um die Arbeit an Bewusstseinszuständen zu begründen. Das immer wieder benannte awakening verspricht, die Welt so zu erkennen, wie sie wirklich ist, ohne auf Instanzen, Institutionen und Expertisen angewiesen zu sein.

Die Rezeption spiritueller Angebote nährt die Hoffnung und den Glauben an die eigene Heilung, die Heilung jedes Individuums und damit der ganzen Welt, an Gemeinschaft und an Verhältnisse, die für alle gleich sind. Wenn jede und jeder seine Konflikte löse, heile die ganze Welt. Mit dem Rekurrieren auf das Authentische, auf Entitäten wie das wahre Selbst, die innere Wahrheit und dem Hinterfragen gesellschaftlicher Glaubenssätze sowie der Ablehnung von Systemen, erscheint alles Politische als das Natürliche. Eine kritische Machtanalyse erscheint obsolet. Wenn es keine gesellschaftlichen Rollen, Strukturen und Ungleichheiten, sondern nur das natürliche Selbst, die Natur des Menschen, das true self, das Authentische und die innere Wahrheit gibt, so kann vermeintlich jede Person ihr Potential entfalten und ihre Realität konstruieren.

Mit dem Titel Gesellschaftlicher Rundumschlag kritisiert eine Alleinerziehende in einem Instagram Posting die Erwartungen der Gesellschaft an ihre Kinder. Was man sich für die eigenen Kinder wünsche, divergiere von dem was die Gesellschaft ihnen ermögliche oder bereitstelle: Die Gesellschaft komme den Bedürfnissen der Menschen nicht nach. Die als gesellschaftlich benannten Probleme werden nicht mehr innerhalb und mit Gesellschaft gelöst, sondern individuell, privatwirtschaftlich und ausserhalb der Gesellschaft. Ebensowenig wie gesellschaftliche Strukturen für alle 
transformiert werden, werden Machtstrukturen betrachtet. Als Individuum, als Dyade oder Familie haben sie die gesellschaftlichen Wege verlassen, um eigene Lösungen zu finden, etwa durch Freilernen, Worldschooling, private Kinderbetreuung und private finanzielle Absicherung.

Man solle seine Arbeit nicht machen, um die Taschen anderer zu füllen, man wolle raus aus dem Hamsterrad und nicht länger Zeit gegen Geld tauschen. Während die Erwerbstätigkeit als Angestellte als Hindernis für das Reisen und als zeitaufwändig diskutiert wird, wird die eigene Selbständigkeit als persönlich erfüllend, als sinnvoll, als Probleme lösend und als Herzensprojekt bezeichnet. Einzelne engagieren sich in privaten sozialen Hilfsprojekten (Charity), die sie selbst initiieren oder unterstützen, oder sie erwägen, Stiftungen zu ihrem Herzensthema zu gründen.

Identität wird als natürlicher innerer Kern verstanden, der zu Tage tritt, wenn gesellschaftliche Rollen und Masken fallen. Kategorien, die Gesellschaft strukturieren, scheinen in einem forcierten Individualismus aufgehoben oder sogar für jeden aufhebbar, wenn man eine Entscheidung treffe, ins Tun komme, Verantwortung übernehme und dem Universum vertraue. Gesellschaft wird dargestellt als etwas gegebenes, einheitliches, aussenstehendes, statisches, fehlerhaftes, nicht reparierbares - etwas das aufzugeben ist, damit das Individuum heilen kann. Das Individuum muss sich optimieren, wenn es ohne staatliche Absicherung leben will. Für die Optimierung des Körpers werden neben Biohacking, gesunder Ernährung und Fitness auch Biotechnologien und Longevity-Technologien begrüsst. Ihre Rentenabsicherung sei, gesünder zu leben, weniger Stress, mehr Sonne, mehr Zeit in der Natur und gesünderes Essen zu haben. Ausserdem sparten sie privat und ihre Arbeit könnten sie im Zweifelsfall noch lange machen. Hinzu kämen die geringeren Lebenshaltungskosten als in Deutschland.

\subsection{Entanglement mit Plattformen und Privatisierung}

Die Online-Selbständigkeit erfordert keine aufwändigen Betriebsmittel oder Investitionen in Anlagen. Geschäftsmodelle wie Amazon FBA (Fulfilment by Amazon) oder die Vermietung von kurzfristig weltweit angemieteten Wohnungen über AirBNB erfordern Tätigkeiten der Datenanalyse und Optimierung von Objektpräsentationen für algorithmisch sortierte Listings. Coaching und Consulting finden via Zoom, WhatsApp, Skype und geschlossenen Facebook- und Telegramgruppen statt. Geschäftsmodelle, die Organisation des Alltags und Kommunikation sind auf datenbasierte Plattformen multinationaler Konzerne angewiesen und nutzen deren Mechanismen zur Generierung von Aufmerksamkeit mit. Strukturen und Regeln der Plattformen sind nicht demokratisch legitimiert, teilweise intransparent. Die Regulierungen von Staaten, wie etwa Visa-Bedingungen, Steuer- und Buchhaltungspflichten, als auch die Regeln von Plattformen gehören zum stetig geteilten Wissensschatz unter Online-Selbständigen. 
Sie werden exploriert, genutzt und ggf. ausgereizt. Regeln zu brechen gehört zum Topos: Alles, was sie in der sterbenden Kultur zum Outsider gemacht habe, habe sie vorbereitet auf eine führende Rolle in der neuen Welt.

Amazon und Facebook werden in den Daten der Studie mehrfach als innovative, ideenreiche und zukunftsgerichtete Infrastrukturen bewundert. Trotz der Ablehnung von Gesellschaft und trotz des Anliegens, den gesellschaftlichen Erwartungen und dem Leistungsdruck nicht mehr nachkommen zu wollen, werden Angebote OnlineSelbständiger auf währungsstarken (Heimat-) Märkten gemacht und wirken in Gesellschaft hinein. Etwa mit Coaching-Angeboten ${ }^{1}$ oder mit dem Geschäftsmodell Amazon FBA, dessen Logistik ortsabhängig erledigt werden muss. Amazon wiederum strebt eine Monopolisierung und Umstrukturierung des Marktes an.

\subsection{Pädagogische Begriffe}

Kernbegriffe von Erziehungs- und Bildungstheorien, wie Selbstbestimmung, Autonomie, Transformation, Wachstum, Persönlichkeitsentwicklung, werden von ortsunabhängig lebenden, online-selbständig Arbeitenden häufig verwendet. Sie beziehen diese in gleicher Weise auf das persönliche wie das ökonomische Wachstum. Wenn man das Versprechen des eigenen Coaching Programms nicht selbst lebe, so ziehe man automatisch auch nicht die Kunden in sein Programm, die man gerne hätte, bzw. wahrscheinlich gar keine. Seinen Traum zu leben bedeute, dass man sich ständig ausdehnen dürfe, und das heisse, dass dem eigenen Wachstum immer Schritte aus der Komfortzone vorangingen. Man müsse mutig sein. Man könne nicht anziehen was man selbst nicht verwirkliche. Stetige Veränderung, Authentizität, Transformation, Wachstum, Persönlichkeitsentwicklung werden anwendungsorientiert konzipiert, das eigene Selbst auf das eigene Business bezogen. Gleichzeitig ist der regelmässige Anspruch, dass Arbeit und wirtschaftliches Wachstum zu Persönlichkeitsentwicklung und persönlichem Wachstum beitrage. Persönlichkeitsentwicklung wird untrennbar mit ökonomischem Wachstum verbunden, was in Beratungsangeboten mit den Begriffen Authentizität und Personal Branding adressiert wird. Transformation wird auf das Individuum bezogen, nicht auf eine wechselseitige Transformation von Individuum und Gesellschaft. Identität entsteht in dieser Logik aus sich selbst heraus, aus dem Erkennen des inneren Kerns.

Schule und Schulpflicht werden teils pragmatisch, weil sie nicht zum ortsunabhängigen Lebensstil passen, teils mit massiver Kritik an der Institution Schule abgelehnt. Schule sei eine Institution, die sich nicht mehr reparieren liesse. Bildung wird so an Herkunft gebunden. Nicht geäussert wird, dass auch Familien Systeme mit Erwartungshaltungen und Rollen bilden. Die Argumentation ortsunabhängig

1 Angebote, die eine Problemlösung auf Ebene des Individuums suchen, wie z. B. selbstbestimmte Geburt, bedürfnisorientierte Erziehung, ortsunabhängig Arbeiten, umweltfreundlich reisen, mit Kindern reisen, Lernen für Kinder mit Matheschwierigkeiten, Zero Waste. 
lebender Familien aber auch die Kommentare der Followerinnen und Follower heben meist auf die Chancen der Kinder ortsunabhängig lebender Familien ab. Ob sie einen Abschluss machen und ob sie sich in die berufliche Welt integrieren könnten, sind oft gesellte Fragen in Kommentaren unter den Instagram Postings. Auch dies ist eine individualistische Perspektive, denn es wird nicht danach gefragt, welche Konsequenzen dies für Schule als Institution, für Gesellschaft, für Demokratie, oder für Schülerinnen und Schüler, die sich nicht abmelden, habe.

Erziehung wird teilweise explizit abgelehnt. Erziehung entfremde den Menschen von seiner natürlichen Bestimmung und seinen Bedürfnissen und mache ihn für Gesellschaft passfähig. Es entfremde Kinder von ihren natürlichen Instinkten und tradiere Angst aus vorausgehenden Generationen. Tradiertes soll abgelegt werden und das Neue soll disruptiv sein. Was vorausgehende Generationen getan haben, um Sicherheit herzustellen, sei nicht mehr relevant. Die stetige Arbeit am Mindest helfe bei der Überwindung des Systems.

\section{Diskussion}

Die dargestellte Praxisform ist zutiefst mit der Entwicklung des Netzes und der Entwicklung neoliberaler Ordnung verwoben. Individuen, die vermeintlich das System hinter sich lassen, steigen tiefer in die Logik eben jenen Systems ein und bauen es weiter aus. Sie übernehmen freiwillig die Anforderungen und Zumutungen, die Neoliberalität an Individuen stellt. Die Praxisform wird hier als post-neoliberal gerahmt, die Staat und staatliche Institutionen als obsolet betrachtet.

\subsection{Geoarbitrage, Automatisierung und Skalierbarkeit und die Ablösung von der Staatsbürgerschaft}

Durch das Modell der Geoarbitrage, durch Geschäftsmodelle, die auf Automatisierung und Skalierbarkeit abzielen, wo Zeit nicht mehr gegen Geld getauscht wird, befreit sich das Individuum. Es gewinnt Zeit für die Familie und die Erfüllung der eigenen Bedürfnisse. Darüber hinaus entledigt es sich zeitaufwendiger und arbeitsintensiver demokratischer (Abstimmungs-)Prozesse. Denn für sich erkannte Probleme des Systems werden nicht etwa innerhalb des Systems und der Gesellschaft oder für alle gelöst. Vielmehr suchen ortsunabhängig lebende Erwachsene und Familien individuelle Lösung für sich selbst. Sie bauen die Strukturen, die sie ablehnen, weiter aus. Die aus einem Online-Business resultierende nicht-automatisierbare Arbeit wird oftmals von anderen erledigt. Sowohl im Angestelltenverhältnis als auch in der Selbständigkeit werde noch Zeit gegen Geld getauscht, während erst ein Online-Business oder ein Investor ein passives Einkommen generiere. Grundlage ist der «Cashflow Quadrant», der vielfach propagiert wird (vgl. Kiyosaki 2014). Erst ein Online-Business schaffe ein eigenes System mit eigenen Regeln. 


\subsection{Die Entwicklung des Netzes und der New Economy}

Während das Modell des ortsunabhängigen Lebens und online selbständigen Arbeitens von seinen Protagonistinnen und Protagonisten als «alternativ oder disruptiv» zum Bestehenden, zur Tradition und zum System bezeichnet wird, kann es auch als Fortsetzung und Weiterführung des bereits Bestehenden interpretiert werden. Fred Turner legt 2006 dar, dass mit dem ökonomischen und sozialen Erfolg des Netzes in den 1990er Jahren bereits seine transformative Kraft evident wurde, die sich auch in dem realisierte, was als New Economy bezeichnet wurde. Diese stellt Anforderungen an Individuen:

«Individuals could no longer count on the support of their employers; they would instead have to become entrepreneurs, moving flexibly from place to place, sliding in and out of collaborative teams, building their knowledge bases and skill sets in a process of constant self-education. The proper role of government in this new environment, many argued, was to pull back, to de-regulate the technology industries that were ostensibly leading the transformations, and,while they were at it, business in general. Proponents of this view included telecommunications executives, high-tech stock analysts, and right-wing politicians.» (Turner 2006, 7)

Die New Economy entstand aus dem Gegenentwurf zu Bürokratie und Technokratie, im Prozess des ökonomischen Erfolgs des World Wide Web und in den neuen Akteursnetzwerken, wie Turner (ebd.) aufzeigt.

Eben diese Anforderungen und Zumutungen der Unternehmen an ihre Arbeitnehmerinnen und Arbeitnehmer scheinen Online-Selbständige nun freiwillig auf sich zu nehmen: Flexibel und mobil zu sein, sich mit den Zielen der eigenen Unternehmung zu identifizieren und stetige Weiterbildung zu leisten. Die Anforderungen und Zumutungen scheinen zu Freiheiten zu werden, wenn sie selbstbestimmt gewählt werden. Im Wunsch, Zeit nicht mehr gegen Geld tauschen zu wollen, wendet das Individuum nun für sich, was Unternehmen ab den 1990er Jahren ihren Mitarbeitenden abverlangten: trotz Zeit basiertem Arbeitsvertrag, zielorientiert geführt zu werden und in kontinuierliche Verbesserungsprozesse eingebunden zu sein.

Die Entwicklung des Netzes und insbesondere des Word Wide Web fand massgeblich ausserhalb (national-)staatlicher Initiative und Steuerung statt (Möller 2019). Die neuen Technologien sollten helfen, die zentralistische Bürokratie des industriellen Zeitalters und die Rationalisierung des sozialen Lebens zu korrodieren und Individuen zu befreien. Bereits früh sind Transnationalität, Antistaatlichkeit, Deregulierung, Ökonomisierung und Privatisierung grundgelegt. Nach Turner (2006) ist dies nicht die Verkehrung einer gegenkulturellen und gemeinschaftsorientierten Bewegung in ihr Gegenteil, sondern in ihr und in den treibenden Akteursnetzwerken bereits angelegt. Er zeichnet das Entstehen eines Techno-Utopismus nach, der bereits im 
Aufkommen einer Gegenkultur in den USA der 1960er Jahre gründet. Ermächtigender Individualismus, individuelle Freiheit, Selbstbestimmung, Freiheit vom Staat, kollaborative Netzwerke von Peers, kollektive Selbstverwaltung, flache Organisationen, spirituelle Gemeinschaften auf dem Lande, Hippietum und Authentizität wurden, ausgehend vom Free Speech Movement, zum Gegenentwurf zu zentralisierter Autorität und Kontrolle des Industriezeitalters und der Technokratie des Kalten Krieges und des Vietnam Krieges, wie sie in Bürokratie und Grossrechnern gesehen wurde. In dieser Utopie sollten Staaten dahinschmelzen und parteienbasierte Politik einer natürlichen Agora im digitalen Raum weichen. In den 1990er Jahren wurde diese Utopie verknüpft mit den aufkommenden Computern, die von Individuen genutzt werden konnten, mit Computernetzen und schliesslich mit dem öffentlichen World Wide Web.

Dass Mitarbeitende das Gleiche wollen wie das Unternehmen, in dem sie angestellt sind, wurde in den 1990er Jahren propagiert, aber bereits auch kritisiert:

«Ich halte es für gefährlich, wenn die Interessen der Mitarbeiter und die der Firma zu sehr miteinander verschmelzen sollen. Ein Unternehmen muss bestimmten ökonomischen Logiken folgen, wenn es überleben will. Und die tragen nicht unbedingt dazu bei, dass Menschen in diesem Unternehmen glücklich werden» (Kühl, 2000).

Im Lebensmodell ortsunabhängig lebender und online selbstständiger Arbeitender wird das Ökonomische vom Persönlichen hingegen nicht mehr getrennt: «Your work is to be totally aligned with yourself» (Vanessa Feils, spiritual coach \& lifestylist, auf Instagram geteilt am 15. März 2021).

\subsection{Entstaatlichung, Sicherheit und Freiheit von staatlicher Regulierung}

Bitcoin basiert auf der Blockchain Technologie, auf der Freiheit von zentralen Instanzen und jeglicher staatlichen Regulierung. Gerard beschreibt Bitcoin als Ideologie, als unreguliert und unsicher:

«Libertarianism is a simple idea: freedom is good and government is bad. The word 'libertarian' originally meant communist and anarchist activities in the 19th-century France. The American right-wing variant starts at fairly normal people who want less bureaucracy and regulation and consider lower taxes are more important than social spending. The seriously ideological ones go rather further - e.g., anarchy-capitalism, the belief in the supremacy of property rights and the complete elimination of the state. American-style libertarians abound on the Internet.» (Gerard 2017, 17f.) 
Allerdings geht es nicht alleine um eine digitale Währung, sondern um die Idee einer dezentralen Gesellschaft (decentralized society). In der Persona der Vorreiter wird diese Bewegung deutlich. Regeln und staatliche Regulierung werden exploriert, ausgereizt und gänzlich abgelehnt. Eigene Regeln zu machen wird offensiv propagiert. Das Ablehnen staatlicher Institutionen und gesellschaftlicher Erwartungen führe ins Natürliche, Freie und Authentische. Tatsächlich führt es in Räume, die unreguliert und unsicher sind. Propagiert und ausgebaut wird ein Zusammenleben, das vermeintlich keine Machtprozesse und Ungleichheit kennt, wo jeder eigene Entscheidungen trifft und eigenverantwortlich handelt. Ein Raum, in dem mach sich selbst bereits einen Vorteil verschaffen konnte und wo man alleine durch das Vertrauen in die eigene Gesundheit, Stärke und Optimierbarkeit abgesichert zu sein scheint. Sicherheit und Freiheit müssen folgerichtig in der Person liegend konstruiert werden, denn sie sind in dieser Logik und in unregulierten Feldern eben nicht relational bestimmt, nicht durch staatliche Institutionen, zentrale Instanzen oder Gesellschaft zugesichert. Sie sind Eigenschaften der Person. Wer Bitcoin oder andere Krypto-Währungen besitzt, gewinnt dadurch, dass andere später einsteigen, denn alleine dadurch steigt der Wert (vgl. Gerard 2017). Durch das eigene Handeln, die Dekonstruktion des Staates und seiner Institutionen und durch Influencing werden diese unregulierten Felder ausgebaut. Private Absicherung durch Assets, Freilernen und Unschooling, gesundheitliche Vorsorge und Behandlung jenseits medizinischer Leitlinien zielen nicht nur auf Privatisierung, sondern auf die gänzliche Freiheit von staatlicher oder institutioneller Regulierung. Digitaler Kapitalismus und Anarcho-Kapitalismus existieren dadurch, dass sie kollektiv hergestellt werden. Auch die Haltung zu Corona zeigt die Überzeugung von der Eigenverantwortung bis hin zur Dekonstruktion des Staates. Selbstbestimmung und Eigenverantwortung stehen im Gegensatz zu Gesellschaft, die als etwas konstruiert wird, das im Aussen stattfindet.

\subsection{Prozesse}

Die Studie zeigt Prozesse auf. Einzelne suchen Alternativen zu Lebenslagen, die als belastend empfunden werden. Genannt werden die schlechte Vereinbarkeit von Familie mit dem Schichtdienst im Krankenhaus, die Gentrifizierung der eigenen Wohnumgebung, die lange Verschuldung für ein Eigenheim, der dunkle nasskalte Winter, Schulangst und eine ADHS-Diagnose, die unflexible Fremdbetreuung der Kinder oder die Wahrnehmung, insbesondere im Alter nicht abgesichert zu sein. Der Einstieg in das ortsunabhängige Leben basiert oft auf der Inspiration durch entsprechende Social Media Accounts und Gruppen. Darüber finden Beratung, Vernetzung und Kommunikation statt. Später wird die Vernetzung und Kommunikation online und offline aufrechterhalten. Narrative und Legitimationsfiguren werden immer wieder reproduziert und reichen bis hin zu antidemokratischen Äusserungen. 
Das Phänomen spannt sich von der Ausblendung sozialer Ungleichheit und Machtverhältnissen bin hin zur pauschaler Ablehnung von Staat, staatlichen Institutionen und Akteuren. Es realisiert sich in einem Glauben an einen Individualismus in seiner radikalen Form und ein Individuum, dessen Lage, Erfolg, Freiheit und Sicherheit alleine in seinen eigenen Entscheidungen, seiner Gesundheit, Fitness, Stärke, seinem Bewusstseinszustand, Gedanken und seiner eigenen Leistung begründet sei. Die eigenen Identitätsbilder konstituieren sich als weltoffen, vorurteilsfrei und natürlich. Die Kommunikation bewegt sich fliessend von einer libertären Haltung bis in antidemokratische Aussagen. Das gesellschaftliche System, die traumatisierende Gesellschaft, den Staat, (staatliche) Regulierung und tradierte Glaubenssätze zu hinterfragen sowie das natürliche Subjekt und individuelle Freiheit zu propagieren, ist anschlussfähig zu extremistisch-rechter Rhetorik. Man sei nicht links, nicht rechts sondern raus aus der Matrix - der Topos konvergiert zum great awakening, der Idee von der Bewusstheit des natürlichen Subjekts. Soweit Spiritualität auf Individualismus zielt, sind «Liebe» Statements oder der Slogan «Leben versus Angst» nicht die Abgrenzung dazu, sondern Teil dessen. Man werde geboren und würde sterben. Dazwischen aber könne man tun was man wolle. Es sei die Gesellschaft, die Regeln generiere, aber da könne man ausbrechen.

\subsection{Digitalisierung und Bildungsinstitutionen}

Digitalisierung ist nicht nur der Prozess der Informatisierung und Datafizierung von Wissensbeständen sondern auch der gesellschaftlichen Transformation. Diese zu gestalten erfordert auch, Digitalisierung und Netzentwicklung als politischen und sozialen Prozess zu verstehen. Staatliche Institutionen werden durch digital kapitalistische Praxisformen herausgefordert. Bildungsinstitutionen kommen in einen Rechtfertigungszwang, wenn Selbstwirksamkeit, Autonomie, Eigenverantwortung und Persönlichkeitsentwicklung ausserhalb von Institutionen vermeintlich besser realisiert werden können als innerhalb.

Neoliberale Ordnung setzt Staat und staatliche Institutionen voraus und erwartet von Staat die Bereitstellung von Infrastruktur und Rechtssicherheit. Digitaler Kapitalismus unterscheidet sich in diesem Punkt und basiert massgeblich auf der Existenz digitaler Plattformen, die Märkte neu strukturieren und staatliche Aufgaben wie Bildung, Infrastrukturen, Mobilität, Gesundheit besser zu erledigen versprechen als staatliche Institutionen. Für den Bereich Bildung siehe zum Beispiel die Google Career Certificates https://grow.google/certificates/. «Die Universitäten als jahrhundertealte Institution der höheren Bildung werden auf bisher nie dagewesene Weise herausgefordert» (Seele 2020, 832). Google verspricht, damit Probleme der Studierenden zu lösen, wie etwa lange Studienzeiten und die Verschuldung durch Studiengebühren. Staatliche Institutionen können insofern nicht digital-kapitalistisch geordnet werden, denn sie erscheinen in dieser Logik schlicht als obsolet. 
Die Aufgabe von Bildungsinstitutionen muss in diesem Zusammenhang sein, die unterschiedlichen Entwicklungslinien der Digitalisierung kritisch zu reflektieren und Alternativen zur digital-kapitalistischen Entwicklung zu imaginieren und zu erproben. Insbesondere aber auch, Demokratie nicht als gegeben vorauszusetzen, sondern stetig neu herzustellen und einzuüben. Die Trennung der Sphären Privat, Öffentlich und Ökonomisch ist nicht naturgegeben, sondern gesellschaftlich geschaffen und muss, wenn eine Gesellschaft an der Trennung (weiterhin) interessiert ist, durch stetiges Tun aufrechterhalten werden. Die Aufgabe von Bildungsinstitutionen ist es, alle mitzunehmen und an demokratischer Arbeit zu beteiligen, gerade auch diejenigen, die sich im digitalen Kapitalismus keinen Vorteil verschaffen können oder wollen.

\section{Fazit}

Vordergründig schafft Digitalisierung schlichtweg die Möglichkeit für einen neuen und ortsunabhängigen Lebensstil. Der Beitrag legte jedoch auch den Ausstieg aus dem System, aus Gesellschaft und staatlichen Institutionen zugunsten des vermehrten Einlassens auf private kommerzielle Akteure sowie das Angewiesensein auf datenbasierte Plattformen dar. Aber nicht nur Plattform-Konzerne, sondern auch Einzelne stellen digitalen Kapitalismus her. Aus der individuellen Freiheit, der Ermächtigung des Individuums und Spiritualität entwickelt sich ein forcierter Individualismus als politische Form. Durch die Orientierung am Privaten und Individuellen wird ein sich zunehmend ausbreitender politischer Raum geschaffen. Das natürliche Individuum wird dem System und der Gesellschaft und das Innere wird dem Äusseren gegenüber gestellt - dem Ideal des autonomen Subjekts folgend. Das vermeintlich Natürliche ist eine kollektiv erzeugte Praxisform. Familie etwa wird nicht als System reflektiert.

Mit ihrem Lebensstil schliessen sich Menschen selbst aus Gesellschaft aus und beabsichtigen, ihre eigene Situation durch das Finden individueller Lösungen, nicht aber durch die Gestaltung gesellschaftliche Strukturen zu ändern. Sie sind privilegiert in dem Sinne, dass sie ihre Sicherheit selbst herstellen, ihren Horizont selbst stabilisieren können: Eine Entscheidungen treffen, alles darauf setzen, ins Tun kommen, ins Vertrauen gehen. Die Flucht aus der leistungsorientierten Gesellschaft erfordert Leistung vom Individuum: Optimierung, Persönlichkeitsentwicklung, körperliche Fitness, Biohacking, Social Media-Arbeit, Arbeit am Mindset, private Absicherung und vieles mehr.

Im Beitrag wurde die Entwicklung der Praxisform in ihrer Kontingenz nachgezeichnet und Zusammenhänge aufgezeigt. Es gelingt hier nicht, jedes aufscheinende Themen theoretisch einzuordnen. Die Einordnung fand anhand einer Entwicklungslinie des Netzes statt. Der Bericht will Prozesse der Digitalisierung und Praktiken der Mediennutzung keinesfalls generalisieren. Neben dieser Entwicklung zeigen sich auch andere, zutiefst demokratische Bestrebungen, die sich zum Beispiel in der Idee 
der Commons realisieren (z. B. Sollfrank 2018; Volkart 2018; Sollfrank, Stalder, und Niederberger 2021). Dies sei hier angemerkt, um Digitalisierung nicht mit digitalem Kapitalismus gleichzusetzen. Es ist eine gesellschaftliche Aufgabe, Formen zu entwickeln, die Gesellschaft und Demokratie stärken. Öffentliche, staatliche Institutionen wie etwa Universitäten, können nicht in digital-kapitalistischer Logik organisiert werden, da diese die Abschaffung staatlicher Institutionen mitbedingt. Sie müssen aus sich heraus andere Formen der Digitalisierung, andere Praktiken und Logiken entwickeln.

\section{Literatur}

Gerard, David. 2017. Attack of the 50 Foot Blockchain: Bitcoin, Blockchain, Etherium and Smart Contracts. First edition. London, England: David Gerard.

Göttlich, Udo. 2010. «Der Alltag der Mediatisierung: Eine Skizze zu den praxistheoretischen Herausforderungen der Mediatisierung des kommunikativen Handelns». In Die Mediatisierung der Alltagswelt, herausgegeben von Maren Hartmann und Andreas Hepp, 23-34. Wiesbaden: VS Verlag für Sozialwissenschaften. https://doi.org/10.1007/978-3-531-92014-6_2.

Hörning, Karl Heinz. 2001. Experten des Alltags. Die Wiederentdeckung des praktischen Wissens. Weilerwist: Velbrück Wissenschaft.

Hörning, Karl H. 2004. «Soziale Praxis zwischen Beharrung und Neuschöpfung. Ein Erkenntnis- und Theorieproblem». In Doing Culture: Neue Positionen zum Verhältnis von Kultur und sozialer Praxis, herausgegeben von Karl H. Hörning und Julia Reuter, 19-39. Bielefeld: transcript Verlag. https://doi.org/10.14361/9783839402436-002.

Kiyosaki, Robert T. 2014. Rich Dad Poor Dad: Was die Reichen ihren Kindern über Geld beibringen. FinanzBuch Verlag.

Kühl, Stefan. 2000. Das Regenmacher-Phänomen. Widersprüche und Aberglauben im Konzept der lernenden Organisation. Campus: Frankfurt a.M.

Möller, Christian. 2019. Kommunikationsfreiheit im Internet: Das UN Internet Governance Forum und die Meinungsfreiheit. Heidelberg: Springer VS. https://doi.org/10.1007/978-3-65827482-5.

Morozov, Evgeny. 2013. To Save Everything, Click Here: The Folly of Technological Solutionism. 1. ed. New York, NY: PublicAffairs.

Nachtwey, Oliver, und Timo Seidl. 2017. Die Ethik der Solution und der Geist des digitalen Kapitalismus (IFS Working Paper11). Institut für Sozialforschung. http://www.ifs.uni-frankfurt.de/wp-content/uploads/IfS-WP-11.pdf.

Turner, Fred. 2006. From counterculture to cyberculture: Stewart Brand, the Whole Earth Network, and the rise of digital utopianism. Chicago: University of Chicago Press. 
Schatzki, Theodore R. 2012. "A Primer on Practices». In Practice-Based Education. Practice, Education, Work and Society, herausgegeben von Joy Higgs, Ronald Barnett, Stephen Billett, Maggie Hutchings, und Franziska Trede, 6:13-26. Rotterdam: SensePublishers. https:// doi.org/10.1007/978-94-6209-128-3_2.

Seele, Peter. 2020. «Greift Google das Hochschulsystem an? Zur Zukunft der Universitäten in Zeiten von KI und Digital-Monopolen, Corona und Home Office». Forschung und Lehre, DHV, 27. Jahrgang. https://www.wissenschaftsmanagement-online.de/beitrag/greift-googledas-hochschulsystem-zur-zukunft-der-universit-ten-zeiten-von-ki-und-digital-11499.

Shaffer, David W. 2006. «Epistemic Frames for Epistemic Games». Computers \& Education 46 (3): 223-34. https://doi.org/10.1016/j.compedu.2005.11.003.

Sollfrank, Cornelia, Hrsg. 2018. Die schönen Kriegerinnen: technofeministische Praxis im 21. Jahrhundert. Wien: Transversal Texts. https://transversal.at/media/femtec_CmBUyFV.pdf.

Sollfrank, Cornelia, Felix Stalder, und Shusha Niederberger, Hrsg. 2021. Aesthetics of the Commons. Bd. 24. The Institute For Contemporary Art Research Series, Zurich University Of The Arts. Zürich: Diaphanes. http://diaphanes.net/titel/aesthetics-of-the-commons-6419.

Staab, Phillip. 2019. Digitaler Kapitalismus: Markt und Herrschaft in der Ökonomie der Unknappheit. Berlin: Suhrkamp.

Volkart, Yvonne. 2018. «Techno-Öko-Feminismus. Unmenschliche Empfindungen in technoplanetarischen Schichten». In Die schönen Kriegerinnen: technofeministische Praxis im 21. Jahrhundert, herausgegeben von Cornelia Sollfrank, 167-202. Wien: Transversal Texts. https://transversal.at/media/femtec_CmBUyFV.pdf.

Zuboff, Shoshana. 2019. «Surveillance Capitalism and the Challenge of Collective Action». New Labor Forum 28(1): 10-29. https://doi.org/10.1177/1095796018819461. 